\title{
A general superdirectivity model for arbitrary sensor arrays
}

\author{
Yong Wang, Yixin Yang, Zhengyao He, Yina Han and Yuanliang Ma*
}

\begin{abstract}
This paper proposes a general model of superdirectivity to provide analytical and closed-form solutions for arbitrary sensor arrays. Based on the equivalence between the maximum directivity factor and the maximum array gain in the isotropic noise field, Gram-Schmidt orthogonalization is introduced and recursively transformed into a matrix form to conduct pre-whitening and matching operations that result in superdirectivity solutions. A Gram-Schmidt mode-beam decomposition and synthesis method is then presented to formally implement these solutions. Illustrative examples for different arrays are provided to demonstrate the feasibility of this method, and a reduced rank technique is used to deal with the practical array design for robust beamforming and acceptable high-order superdirectivity. Experimental results that are provided for a linear array consisting of nine hydrophones show the good performance of the technique. A superdirective beampattern with a beamwidth of $48.05^{\circ}$ in the endfire direction is typically achieved when the inter-sensor spacing is only $0.09 \lambda$ ( $\lambda$ is the wavelength), and the directivity index is up to $12 \mathrm{~dB}$, which outperforms that of the conventional delay-and-sum counterpart by $6 \mathrm{~dB}$.
\end{abstract}

Keywords: Gram-Schmidt orthogonalization; High-order superdirectivity; Mode-beam decomposition and synthesis; Optimal beamforming; Sonar signal processing

\section{Introduction}

Sensor array signal processing has played a significant role in many diverse application areas, including sonar, radar, audio engineering, and wireless communication [1]. As an important topic in sensor array signal processing, superdirectivity has received considerable attention for decades [2-6], because it can provide a significant potential for sensor arrays to enhance their performance, such as in terms of angular resolution, bearing estimation accuracy, noise suppression ability, and reduction of array aperture. A wideband constant beamwidth and shortened near-field zone can also be conveniently obtained. However, open problems concerning superdirectivity exist in error sensitivity or performance robustness. Although the robustness problem is inevitable for superdirective arrays, this requires an in-depth study to determine a mechanism to decrease the effect of errors as much as possible.

Different researchers have investigated this problem in different aspects and correspondingly proposed many approaches. The classical optimal method was constrained

\footnotetext{
* Correspondence: ylma@nwpu.edu.cn

School of Marine Science and Technology, Northwestern Polytechnical University, Xi'an 710072, China
}

to improve robustness by implementing the diagonal loading [7] and white noise gain constraint techniques [8]. Although they are widely used, the associated parameters in these techniques are not easily determined. Actually, the above-mentioned two techniques belong to the class of regularization-based techniques that can make trade-offs between directivity and robustness by adding a proper regularization parameter to all the diagonal elements of the noise covariance matrix. A detailed study of the regularization-based techniques was presented in [9], and several criteria were proposed for selecting regularization parameters. However, this approach demands a pre-specified distribution of errors that may not be available in practice. Broadband beamformers with superdirectivity robust against gain and phase errors in the microphone array characteristics were designed in [10] in which several properly formulated cost functions were used. An alternative simplified procedure based on the similar principle was presented in [11] to design robust superdirective broadband beamformers for equally spaced linear arrays. Moreover, an optimum superdirective beamformer was designed in [12] by maximizing the generalized directivity that was 
computed using the probability density functions of the array errors. These methods provide good insights into superdirectivity, but they also require a priori knowledge of errors. This problem also exists in the superdirective method proposed in [13]. Many other analyses on the robustness problem were presented in [14-16]. As for the general model of superdirectivity, a model for generalized acoustic sensors of arbitrary order was formulated in [17]. However, this model was based on Taylor series approximation aside from a closed-form solution. Vector sensor array theory also suffers from this limitation of Taylor series approximation [18-20]. These achievements are valuable for us to obtain an improved understanding on how to solve this problem. However, a general model that provides an analytical and closed-form solution is still lacking to accurately calculate a superdirective beamformer and allow the decomposition of the beampattern into components with different error sensitivities for robust implementation.

Answers were provided in [21, 22] for the abovementioned problem in which an eigen-beam decomposition and synthesis (EBDS) model was formulated. However, the array shapes were restricted to a circular form. The eigen-beam decomposition becomes feasible because of the circulant property of the data covariance matrix of circular arrays. This condition is not the case for sensor arrays with other geometries, and a new model that can be generally applicable to arbitrarily shaped arrays is therefore required. This study presents proof that a Gram-Schmidt mode-beam decomposition and synthesis (GSMDS) method can work as a general model for the problem at hand.

The newly developed model is intended to be used in designing a linear array. This type of linear array is particularly important because among all array geometries, it is best suited for superdirectivity for the given array size and number of sensors when the beam is steered toward the endfire direction [23]. Several illustrative examples will be provided in which this model will be applied not only to linear arrays but also to other types of arrays. An important property that is similar to the circular array case will be shown in which low-order mode-beams are robust, whereas high-order ones become sensitive to configuration and channel response errors. Therefore, a reduced-rank technique can be readily used to obtain a robust design, which truncates unsatisfactory high-order mode-beams and retains robust low-order ones. Experiments for a linear array will be conducted to verify the theoretical results.

The rest of this paper is organized as follows. The "Background" section provides a brief description of optimal beamforming theory. The "Gram-Schmidt modebeam decomposition and synthesis" section presents the principles of superdirectivity based on the GSMD and derives the solutions of the optimal weighting vector, beampattern synthesis equation, directivity synthesis equation, and robustness parameter. The "Design examples" section presents illustrative design examples for different arrays, and the "Comparisons with the DAS and MVDR methods" and "Comparisons with the regularization-based methods" sections compare the proposed method with other related methods. The "Experimental results" section provides the experimental results for a linear array. Finally, the "Conclusions" section presents the conclusions.

\section{Background}

Consider an $\mathrm{N}$-sensor array with an arbitrary geometry, and assume all of the sensors are omnidirectional. A unit-magnitude plane wave impinges from direction $(\theta, \phi)$, where $\theta$ and $\phi$ denote elevation and azimuth angles, respectively. The pressure received by the $n$th sensor is as follows [1]:

$$
a_{n}(\theta, \phi)=e^{-i \mathbf{k}^{\mathrm{T}} \mathbf{r}_{n}}
$$

where $i=\sqrt{-1}$ and $\mathbf{r}_{n}$ is the position vector of the $n$th sensor. The wave-vector is $\mathbf{k}=-k[\sin \theta \cos \phi, \sin \theta \sin \phi$, $\cos \theta]^{\mathrm{T}}$, where $k=2 \pi / \lambda$, the symbol $\lambda$ indicates the wavelength, and the superscript $\mathrm{T}$ indicates the transpose. The manifold vector is

$$
\mathbf{a}(\theta, \phi)=\left[a_{0}(\theta, \phi), a_{1}(\theta, \phi), \cdots, a_{N-1}(\theta, \phi)\right]^{\mathrm{T}} .
$$

The beampattern is defined as

$$
B\left(\theta_{0}, \phi_{0}, \theta, \phi\right)=\mathbf{w}^{\mathrm{H}}\left(\theta_{0}, \phi_{0}\right) \mathbf{a}(\theta, \phi),
$$

where the superscript $\mathrm{H}$ indicates the Hermitian transpose, the vector $\mathbf{w}$ is the weighting vector, and $\left(\theta_{0}, \phi_{0}\right)$ is the preset steering direction.

The concept of superdirectivity is introduced in [24], which states that a small sensor array can outperform its delay-and-sum (DAS) counterpart and provide high directivity. After the development of optimal sensor array theory, the maximum directivity factor (DF) of a sensor array is equivalent to the maximum array gain (AG) when the array is used in an isotropic noise field [25]. This relationship can be expressed as [21]

$$
\mathrm{DF}_{\max }=\mathrm{AG}_{\text {max } \mid \text { isotropic noise, }}
$$

where AG is defined as Eq. (5) $[1,21]$ :

$$
\mathrm{AG}=\frac{\mathrm{SNR}_{\mathrm{o}}}{\mathrm{SNR}_{\mathrm{i}}}=\frac{\left|\mathbf{w}^{\mathrm{H}}\left(\theta_{0}, \phi_{0}\right) \mathbf{a}(\theta, \phi)\right|^{2}}{\mathbf{w}^{\mathrm{H}} \mathbf{R}_{n n} \mathbf{w}} .
$$

Note that the logarithmic version of DF $(10 \log D F)$ is defined as the directivity index (DI). The symbols $S N R_{\mathrm{o}}$ and $S N R_{\mathrm{i}}$ in Eq. (5) are the output and input signal-to-noise ratios, respectively. The matrix $\mathbf{R}_{n n}$ is the normalized noise 
covariance matrix composed of noise correlation coefficients, which is defined as

$$
\mathbf{R}_{n n}=\left[\begin{array}{cccc}
\rho_{00} & \rho_{01} & \cdots & \rho_{0, N-1} \\
\rho_{10} & \rho_{11} & \cdots & \rho_{1, N-1} \\
\vdots & \vdots & \vdots & \vdots \\
\rho_{N-1,0} & \rho_{N-1,1} & \cdots & \rho_{N-1, N-1}
\end{array}\right] .
$$

The optimal weighting vector for the maximum AG is [1]

$$
\mathbf{w}_{\mathrm{opt}}=\mathbf{R}_{n n}^{-1} \mathbf{a}\left(\theta_{0}, \phi_{0}\right) .
$$

If the sensor array is placed in an isotropic noise field, and the inter-sensor spacing is equal to or much larger than a half-wavelength, its conventional DAS beamformer will be optimal in AG because the noise covariance matrix is an identity matrix in this case. The optimization of AG in the isotropic noise field will result in superdirectivity when the spacing becomes small in wavelength. Therefore, superdirectivity can be formulated in the context of AG optimization under the assumptions that the spacing is small and the noise field is isotropic.

The problem is how to determine a general model to accurately solve the weighting vector of superdirectivity. Accuracy is desirable because superdirectivity is error sensitive, and calculation errors can destroy the superdirective property. As an alternative, a two-step approach can help us determine the general model with analytical and closed-form expressions, as shown in the following.

\section{Gram-Schmidt mode-beam decomposition and synthesis}

As a useful algorithm, GS orthogonalization has been widely used in array signal processing. Specifically, the GS orthogonalization technique has been proved to be suitable for least-squares estimation because of its good robustness [26], and this technique has also shown superior performance in arithmetic efficiency, stability, and convergence times over other adaptive algorithms for adaptive cancelation [27, 28]. Moreover, the GS-based algorithm was used to synthesize desired beampattern for different arrays [29], and an optimizing approach based on the GS orthogonalization was applied in [30] to optimize loudspeaker and microphone configurations for sound reproduction systems.

It is clear that applying the GS orthogonalization in array signal processing is not novel, but this technique has not yet been used to develop a general model of superdirectivity for arbitrary sensor arrays. In this study, the solutions of superdirectivity will be accurately expressed in full closed-form based on the GS orthogonalization, and the derived Gram-Schmidt mode-beam decomposition and synthesis (GSMDS) superdirectivity model will facilitate the implementation of high-order superdirectivity in practice. See more details in the following sections.

\subsection{Matrix form of the optimal weighting vector}

The mechanism of AG optimization was explained in [31]. An optimal array processor can be implemented in two steps: pre-whitening and matching. Initially, the received data vector will be sent to a pre-whitening processor that pre-whitens its contained noise data vector so that the noise power can be largely decreased. The larger correlation between channels, the more will be the reduction of the noise power. A matching operation follows, which matches the data output modified by the pre-whitening processor to a signal steering vector, and then the received signals impinging from the desired direction are added coherently, whereas the noises are added incoherently. Therefore, the output signal-tonoise ratio can be greatly improved and the processing gain is much higher than the simple DAS method that does not pre-whiten the noises. This process is shown in Fig. 1 in which the received data vector $\mathbf{X}$ contains the signal vector $\mathbf{S}$ and noise vector $\mathbf{U}$. The vector $\mathbf{V}$ is the pre-whitened output noise vector, and $\boldsymbol{\varepsilon}$ is the normalized version of $\mathbf{V}$. The entries of both $\mathbf{U}$ and $\mathbf{V}$ are supposed to be narrow band complex analytic functions, the matrix $\mathbf{C}$ performs the orthogonal transform, and the relation $\mathbf{V}=\mathbf{C U}$ holds. Therefore, $V_{j}$ can be expressed as a linear combination of the $j$ terms of $U_{h}(h=0,1, \ldots . ., j)$, i.e.,

$$
V_{j}=c_{j 0} U_{0}+c_{j 1} U_{1}+\cdots+c_{j j} U_{j}=\sum_{h=0}^{j} c_{j h} U_{h}
$$

The pre-whitening process can be performed with the GS orthogonal transform, and $\mathbf{V}$ can be recursively derived from $\mathbf{U}$ :

$$
\begin{aligned}
& V_{k}=U_{k}-\sum_{j=0}^{k-1} \frac{\left\langle U_{k}, V_{j}\right\rangle}{\left\langle V_{j}, V_{j}\right\rangle} V_{j}, \\
& \varepsilon_{k}=\frac{V_{k}}{\left|V_{k}\right|} \quad(k=0,1, \cdots, N-1),
\end{aligned}
$$

where $\mathbf{V}$ is viewed as an orthogonal basis for the $N \times 1$ complex space $\mathbf{C}^{N}$ formed by the noise data vector $\mathbf{U}$. The modulus of the vector component $V_{k}$ is $\left|V_{k}\right|=$ $\sqrt{\left\langle V_{k}, V_{k}\right\rangle}$, where $\langle\cdot, \cdot\rangle$ indicates the inner product that expresses the cross correlation between the related vector components.

Substituting Eq. (8) into Eq. (9) and using some mathematical derivations yield 


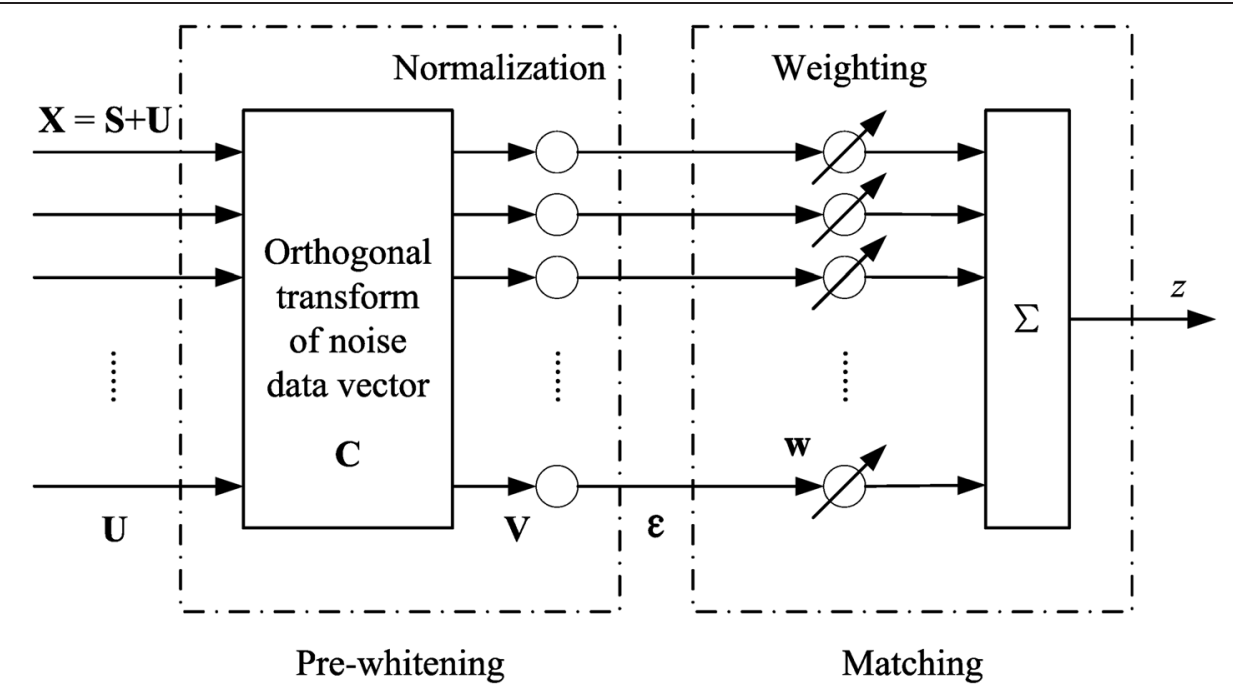

Fig. 1 Block diagram of the optimal array processing expressed as pre-whitening and matching

$$
c_{k i}=\left\{-\sum_{j=i}^{k-1}\left\{\frac{c_{j i}}{X_{j}}\left(\sum_{h=0}^{j} c_{j h} \rho_{k h}\right)\right\} \quad \text { if } i \leq k \leq N-1 \text { and } h, i, j \leq(k-1),\right.
$$

where

$$
\begin{aligned}
X_{j} & =\left\langle\left(\sum_{h=0}^{j} c_{j h} U_{h}\right),\left(\sum_{m=0}^{j} c_{j m} U_{m}\right)\right\rangle \\
& =\sum_{h=0}^{j} \sum_{m=0}^{j} c_{j h} c_{j m}\left\langle U_{h}, U_{m}\right\rangle \\
& =\sum_{h=0}^{j} \sum_{m=0}^{j} c_{j h} c_{j m} \rho_{h m}
\end{aligned}
$$

and $\rho_{i j}=\left\langle U_{i}, U_{j}\right\rangle$ are the correlation coefficients. These equations are similar to the results presented in [29] in which array synthesis is the main focus.

Utilizing the recursive deduction for $c_{k i}$ provides the matrix $\mathbf{C}$ as

$$
\mathbf{C}=\left[\begin{array}{llllll}
\mathbf{C}_{0} & \mathbf{C}_{1} & \cdots & \mathbf{C}_{k} & \cdots & \mathbf{C}_{N-1}
\end{array}\right]^{\mathrm{T}}
$$

with $\mathbf{C}_{k}=\left[\begin{array}{lllllll}c_{k 0} & c_{k 1} & \cdots & c_{k k} & 0 & \cdots & 0\end{array}\right]^{\mathrm{T}}$. Each entry of this lower triangular matrix will be completely determined by the noise correlation coefficients. For the isotropic noise field, the correlation coefficients are

$$
\rho_{i j}=\frac{\sin \left(2 \pi d_{i j} / \lambda\right)}{2 \pi d_{i j} / \lambda},
$$

where $d_{i j}$ is the spacing between the $i$ th and $j$ th sensors.
Because the vector $\mathbf{V}$ is pre-whitened, the following is obtained:

$$
\left\langle\mathbf{V}, \mathbf{V}^{\mathrm{T}}\right\rangle=\mathbf{D}^{2}
$$

where $\mathbf{D}=\operatorname{diag}\left\{\left|V_{0}\right|,\left|V_{1}\right|, \cdots,\left|V_{N-1}\right|\right\}, \quad \mathbf{D}^{2}=\mathbf{D} \cdot \mathbf{D}$, and $\operatorname{diag}\{\cdot\}$ indicate a square matrix with the elements of its arguments on the diagonal.

Substituting the relation $\mathbf{V}=\mathbf{C U}$ into Eq. (14) yields

$$
\mathbf{C}\left\langle\mathbf{U}, \mathbf{U}^{\mathrm{T}}\right\rangle \mathbf{C}^{\mathrm{T}}=\mathbf{C R}_{n n} \mathbf{C}^{\mathrm{T}}=\mathbf{D}^{2},
$$

which then obtains

$$
\mathbf{R}_{n n}^{-1}=\mathbf{C}^{\mathrm{T}} \mathbf{D}^{-2} \mathbf{C}
$$

It is now clear that the inverse of matrix $\mathbf{R}_{n n}$ can be calculated analytically based on the GS orthogonalization. Actually, the inverse of matrix $\mathbf{R}_{n n}$ can also be calculated using the eigen-decomposition, as was the case for circular arrays in [21, 22], but the eigenvalues and eigenvectors in relation to other array geometries cannot be expressed in closed-form and computed analytically. Therefore, the GS-based decomposition is more advantageous than the eigen-decomposition for computing the inverse of the normalized noise covariance matrix.

The optimal weighting vector in Eq. (7) can be modified to

$$
\mathbf{w}_{\mathrm{opt}}=\mathbf{C}^{\mathrm{T}} \mathbf{D}^{-2} \mathbf{C} \cdot \mathbf{a}\left(\theta_{0}, \phi_{0}\right)
$$

where the matrix $\mathbf{D}^{-2}$ performs the normalization of the noise input. The matching vector is 


$$
\mathbf{w}_{\text {match }}=\mathbf{C a}\left(\theta_{0}, \phi_{0}\right) \text {. }
$$

The elements of this vector are strictly marched with the data output modified by the pre-whitening processor, which can make the signals to be added coherently. The beampattern and other array performance parameters, such as AG, can be directly deduced from this solution.

The general model of superdirectivity will be developed based on the above results in the following section.

\subsection{Mode-beam decomposition and synthesis}

Substituting Eq. (17) into Eq. (3) gives the superdirective beampattern as

$$
\begin{aligned}
B\left(\phi_{0}, \theta_{0}, \phi, \theta\right) & =\mathbf{w}_{\mathrm{opt}}^{\mathrm{H}} \mathbf{a}(\theta, \phi) \\
& =\left[\mathbf{C} \cdot \mathbf{a}\left(\theta_{0}, \phi_{0}\right)\right]^{\mathrm{H}} \cdot \mathbf{D}^{-2} \cdot[\mathbf{C} \cdot \mathbf{a}(\theta, \phi)] \\
& =\sum_{k=0}^{N-1} b_{k}\left(\theta_{0}, \phi_{0}, \theta, \phi\right)
\end{aligned}
$$

The $k$ th-order mode-beam in the above equation is

$$
b_{k}\left(\theta_{0}, \phi_{0}, \theta, \phi\right)=\frac{1}{\lambda_{k}} \cdot E_{k}^{*}\left(\theta_{0}, \phi_{0}\right) E_{k}(\theta, \phi),
$$

where

$$
E_{k}(\theta, \phi)=\mathbf{C}_{k}^{\mathrm{T}} \mathbf{a}(\theta, \phi)
$$

and

$$
\begin{aligned}
\lambda_{k} & =\left|V_{k}\right|^{2}=\left\langle V_{k}, V_{k}\right\rangle \\
& =\mathbf{C}_{k}^{\mathrm{T}} \mathbf{R}_{n n} \mathbf{C}_{k} \quad(k=0,1, \cdots, N-1),
\end{aligned}
$$

and the superscript asterisk indicates complex conjugation. The DF (or the AG because they are equal in this case) is derived as

$$
\begin{aligned}
D F & =\left[\mathbf{C} \cdot \mathbf{a}\left(\theta_{0}, \phi_{0}\right)\right]^{\mathrm{H}} \mathbf{D}^{-2}\left[\mathbf{C} \cdot \mathbf{a}\left(\theta_{0}, \phi_{0}\right)\right] \\
& =\sum_{k=0}^{N-1}\left|E_{k}\left(\theta_{0}, \phi_{0}\right)\right|^{2} / \lambda_{k} \\
& =\sum_{k=0}^{N-1} Q_{k}
\end{aligned}
$$

with

$$
Q_{k}=\left|E_{k}\left(\theta_{0}, \phi_{0}\right)\right|^{2} / \lambda_{k}
$$

with the use of Eqs. (5), (16), and (17). The symbol $Q_{k}$ is the AG or DF of the $k$ th-order mode-beam.

The sensitivity function (SF) is always used to measure the robustness of beamformers. A large SF corresponds to poor robustness, implying that the errors that can be tolerated are small. The SF is defined as follows [1]:

$$
T=\|\mathbf{w}\|^{2},
$$

where $\|\cdot\|$ indicates the Euclidean norm. Substitution of Eq. (17) into Eq. (25) yields the total SF:

$$
\begin{aligned}
S F= & \mathbf{E}^{\mathrm{H}}\left(\theta_{0}, \phi_{0}\right) \mathbf{D}^{-2} \hat{\mathbf{C}} \mathbf{D}^{-2} \mathbf{E}\left(\theta_{0}, \phi_{0}\right) \\
= & \sum_{k=0}^{N-1} \sum_{k^{\prime}=0}^{N-1} \frac{1}{\lambda_{k} \lambda_{k^{\prime}}} E_{k}^{*}\left(\theta_{0}, \phi_{0}\right) E_{k^{\prime}}\left(\theta_{0}, \phi_{0}\right) \hat{\mathbf{C}}_{k^{\mathrm{T}}}^{\mathrm{T}} \hat{\mathbf{C}}_{k} \\
= & \sum_{k=0}^{N-1} \underbrace{\frac{1}{\lambda_{k}^{2}}\left|E_{k}\left(\theta_{0}, \phi_{0}\right)\right|^{2} \hat{\mathbf{C}}_{k}^{\mathrm{T}} \hat{\mathbf{C}}_{k}}_{T_{k}} \\
& +\sum_{k=0}^{N-1} \sum_{k^{\prime}=0, k \neq k^{\prime}}^{N-1} \frac{1}{\lambda_{k} \lambda_{k^{\prime}}} E_{k}^{*}\left(\theta_{0}, \phi_{0}\right) E_{k^{\prime}}\left(\theta_{0}, \phi_{0}\right) \hat{\mathbf{C}}_{k^{\prime}}^{\mathrm{T}} \hat{\mathbf{C}}_{k},
\end{aligned}
$$

where $\mathbf{E}\left(\theta_{0}, \phi_{0}\right)=\left[E_{0}\left(\theta_{0}, \phi_{0}\right) \quad E_{1}\left(\theta_{0}, \phi_{0}\right) \quad \cdots \quad E_{N-1}\left(\theta_{0}\right.\right.$, $\left.\left.\phi_{0}\right)\right]^{\mathrm{T}}, \hat{\mathbf{C}}=\mathbf{C C}^{\mathrm{T}}=\left[\begin{array}{llllll}\hat{\mathbf{C}}_{0} & \hat{\mathbf{C}}_{1} & \cdots & \hat{\mathbf{C}}_{k} & \cdots & \hat{\mathbf{C}}_{N-1}\end{array}\right]^{\mathrm{T}}$. The SF of the $k$ th-order mode-beam is $T_{k}=\lambda_{k}^{-2}\left|E_{k}\left(\theta_{0}, \phi_{0}\right)\right|^{2}$. $\hat{\mathbf{C}}_{k}^{\mathrm{T}} \hat{\mathbf{C}}_{k}$, which is obtained by combining Eqs. (20) and (25). It is observed that $\lambda_{k}$ is directly related to the total SF and the SF of each mode-beam, which can be used to measure the robustness. The larger the value of $\lambda_{k}$, the lesser is the SF of the $k$ th-order mode-beam, meaning the smaller is the sensitivity to errors. However, the total $\mathrm{SF}$ is not equal to the sum of the SFs of mode-beams because the matrix $\hat{\mathbf{C}}$ is not an orthogonal matrix, which is different from that of the EBDS model. Since the expression of total SF is somewhat complex and no superposition property similar to that of the EBDS model exists, the parameter $\lambda_{k}$ will be used in this paper to measure the robustness instead of the SF.

From Eqs. (19) and (23), it is clear that the overall beampattern and DF of an $N$-sensor superdirective array can be decomposed into $N$ mode-beams and their associated DFs, respectively. These properties of the GSMDS method are similar to those of the EBDS method. The value of $\lambda_{k}$ in the low-frequency range for specific arrays (e.g., linear arrays) will be proved to decrease with an increase in its order, which means that the error sensitivity of this modebeam increases. Therefore, a reduced-rank treatment that requires truncation of unsatisfactory high-order mode-beams and retention of robust low-order ones can be straightforwardly used to synthesize robust superdirective beampatterns. The maximum order of the required mode-beams in Eqs. (19) and (23), which will be denoted as $K$ in the following, should then be smaller than or equal to $N-1$. Mode-beam extraction for a practical case of error distribution can be conducted through experimental measurements or computer simulations.

It is noteworthy that the optimal solution that provides maximum processing gain in different noise fields can also be derived using the above-mentioned 


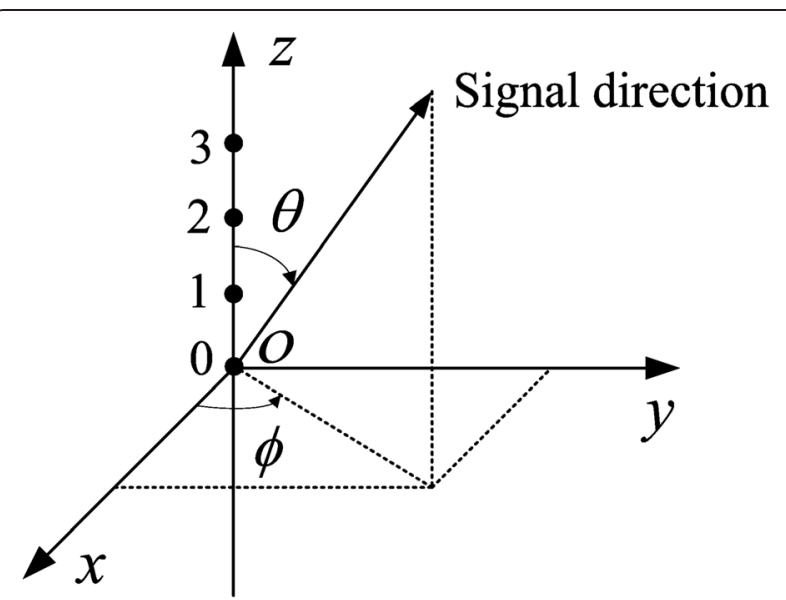

Fig. 2 Coordinates of a four-sensor uniform linear array

procedure. However, the noise correlation coefficients between array elements cannot be always determined analytically, and sometimes, they should be estimated using in situ measurements. In other words, the general solution of the optimal array signal processing can be expressed analytically or adjusted adaptively. Because the superdirectivity is an important topic as mentioned previously, this study is only focused on the isotropic noise field, and the analytical and close-form solutions are directly derived.

For the purpose of clearly showing the performance of the GSMDS model, it is necessary to study the actual DF in the presence of errors.

In actual systems, sensor mismatches will affect the manifold and corrupt the beampattern. For simplicity, the present study takes into account just the gain and phase errors.

The gain and phase errors of the $k$ th sensor are assumed to be $g_{k}$ and $\psi_{k}$, respectively, and the actual received pressure will be

$$
\begin{aligned}
\tilde{a}_{k}(\theta, \phi) & =A_{k} a_{k}(\theta, \phi) \\
& =\left(1+g_{k}\right) e^{-i \psi_{k}} a_{k}(\theta, \phi) .
\end{aligned}
$$

Then, the beampattern in Eq. (3) changes to

$$
\tilde{B}(\theta, \phi)=\mathbf{w}^{\mathrm{H}}[\mathbf{A a}(\theta, \phi)]
$$

In the presence of sensor gain and phase errors, the directivity of the array will inevitably degrade. From the above discussion, the actual DF of the $k$ th-order mode-beam can be defined as

$$
\begin{aligned}
\tilde{Q}_{k} & =\frac{\left|\tilde{b}_{k}\left(\theta_{0}, \phi_{0}\right)\right|^{2}}{\frac{1}{4 \pi} \int_{0}^{2 \pi} \int_{0}^{\pi}\left|\tilde{b}_{k}(\theta, \phi)\right|^{2} \sin \theta d \theta d \phi} \\
& =\frac{\left|\frac{1}{\lambda_{k}} E_{k}^{*}\left(\theta_{0}, \phi_{0}\right) \tilde{E}_{k}\left(\theta_{0}, \phi_{0}\right)\right|^{2}}{\frac{1}{\lambda_{k}^{2}}\left|E_{k}\left(\theta_{0}, \phi_{0}\right)\right|^{2}\left[\frac{1}{4 \pi} \int_{0}^{2 \pi} \int_{0}^{\pi} \tilde{E}_{k}(\theta, \phi) \tilde{E}_{k}^{*}(\theta, \phi) \sin \theta d \theta d \phi\right]} \\
= & \frac{\left|\tilde{E}_{k}\left(\theta_{0}, \phi_{0}\right)\right|^{2}}{\mathbf{C}_{k}^{\mathrm{H}} \tilde{\mathbf{R}}_{n n} \mathbf{C}_{k}}
\end{aligned}
$$

where $\tilde{\mathbf{R}}_{n n}$ denotes the practical noise covariance matrix, which is

$$
\begin{aligned}
\tilde{\mathbf{R}}_{n n} & =\frac{1}{4 \pi} \int_{0}^{2 \pi} \int_{0}^{\pi} \tilde{\mathbf{a}}(\theta, \phi) \cdot \tilde{\mathbf{a}}^{\mathrm{H}}(\theta, \phi) \sin \theta \mathrm{d} \theta \mathrm{d} \phi \\
& =\mathbf{A R}_{n n} \mathbf{A} .
\end{aligned}
$$

If there are no errors, Eq. (29) will be equal to Eq. (24). The average of the actual DF of the $k$ th-order mode-beam is defined as

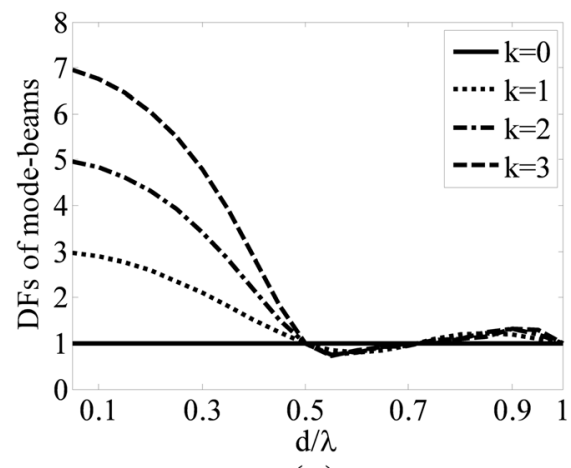

(a)

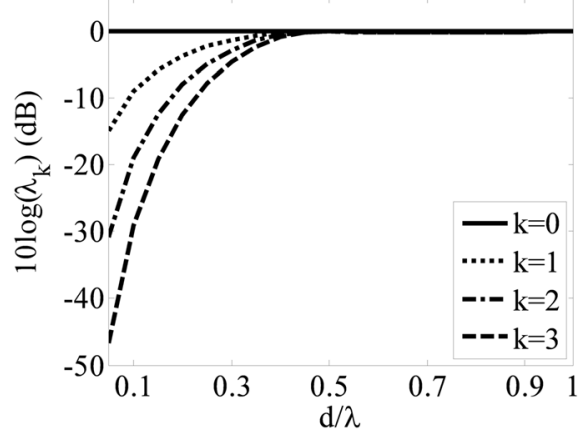

(b)

Fig. 3 DFs and levels of robustness of the 0th- to 3rd-order mode-beams for the four-sensor uniform linear array. a DFs. $\mathbf{b}$ Levels of robustness 


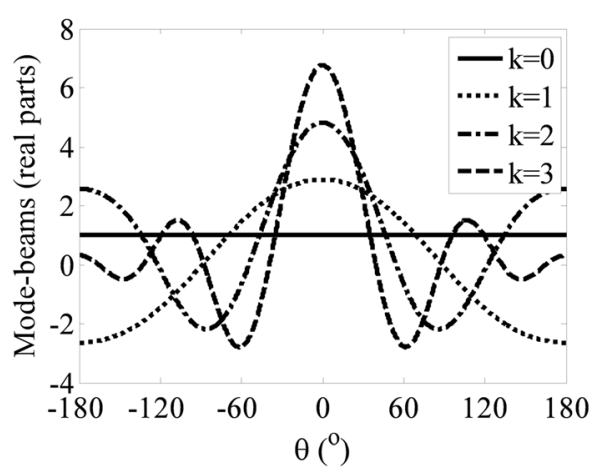

(a)

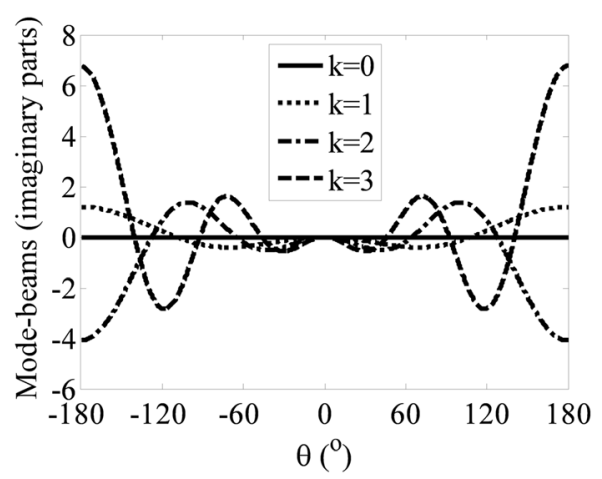

(b)

Fig. 4 The 0th- to 3rd-order mode-beams of the four-sensor uniform linear array at $d / \lambda=0.1$. a Real parts. b Imaginary parts

$$
\bar{Q}_{k}=E\left\{\frac{\left|\tilde{E}_{k}\left(\theta_{0}, \phi_{0}\right)\right|^{2}}{\mathbf{C}_{k}^{\mathrm{H}} \tilde{\mathbf{R}}_{n n} \mathbf{C}_{k}}\right\} .
$$

Actually, the sensor errors also increase the signal gain, which will enhance the DF. However, this additional benefit can be neglected in view of the degradation caused by errors [3]. Thus, for obtaining a conservative estimate, the average of the actual DF will be defined as

$$
\bar{Q}_{k} \approx \frac{\left|E_{k}\left(\theta_{0}, \phi_{0}\right)\right|^{2}}{\mathbf{C}_{k}^{\mathrm{H}} \overline{\mathbf{R}}_{n n} \mathbf{C}_{k}}
$$

where the derivation of $\overline{\mathbf{R}}_{n n}=E\left\{\tilde{\mathbf{R}}_{n n}\right\}$ and its calculation method can be found in [13]. The average DFs of other beamforming methods can be similarly defined as

$$
\overline{D F}=E\left\{\frac{\left|\mathbf{w}^{\mathrm{H}} \tilde{\mathbf{p}}\left(\theta_{0}, \phi_{0}\right)\right|^{2}}{\mathbf{w}^{\mathrm{H}} \tilde{\mathbf{R}}_{n n} \mathbf{w}}\right\} \approx \frac{\left|\mathbf{w}^{\mathrm{H}} \mathbf{p}\left(\theta_{0}, \phi_{0}\right)\right|^{2}}{\mathbf{w}^{\mathrm{H}} \overline{\mathbf{R}}_{n n} \mathbf{w}} .
$$

\section{Design examples}

Some design examples for different arrays are provided in this section to demonstrate the feasibility of the GSMDS model.

\subsection{Linear arrays}

Linear arrays for superdirectivity have been extensively investigated [11, 24, 32-36]. An endfire linear array has

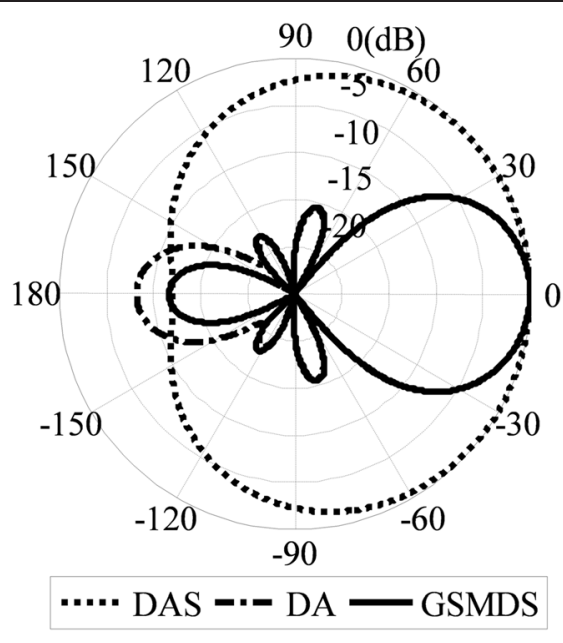

(a)

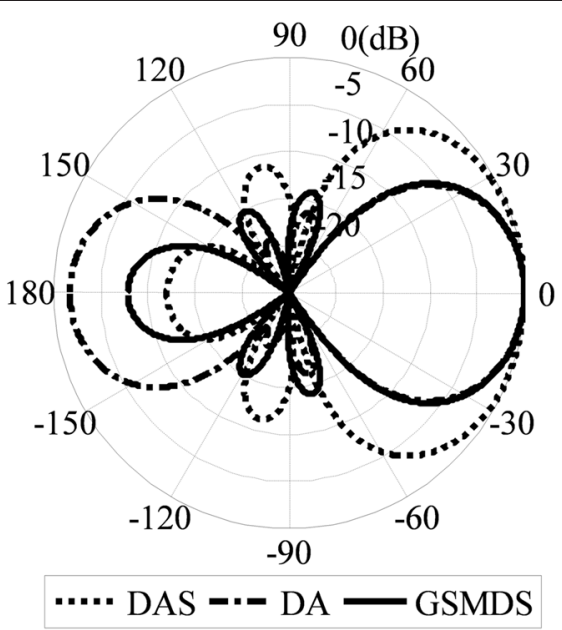

(b)

Fig. 5 Synthesized beampatterns of the four-sensor uniform linear array with the use of different models at $d / \lambda=0.1$ and 0.3 . a $d / \lambda=0.1$. b $d / \lambda=0.3$ 


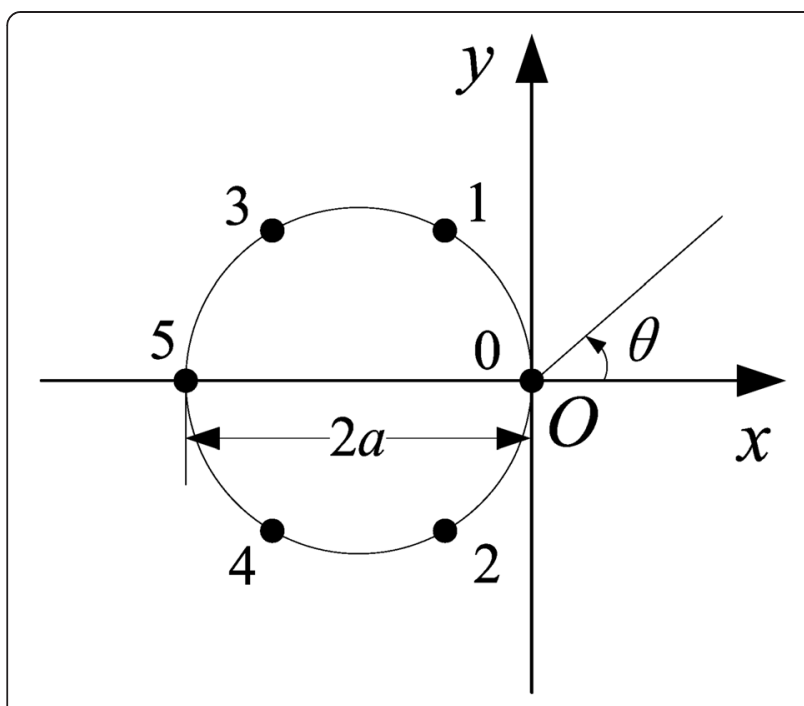

Fig. 6 Coordinates of a six-sensor uniform circular array

been proven to possess the highest superdirectivity for a given number of sensors, and its DI approaches $20 \log N$ $(\mathrm{dB})$ when the inter-sensor spacing becomes infinitely small $[2,23]$, where $N$ is the number of sensors. For this particular array, a difference approximation (DA) model exists [35]. It provides promising calculation accuracy when the spacing and overall length of the array are small. However, this model is difficult to implement in practice because it is highly error sensitive, which is why only first-order (vector sensor) or second-order linear arrays (at most) exist thus far. The newly developed general model should be tested if it can significantly relax restrictions on the spacing and overall size (and thus the sensor number $N$ ).

Consider an equally spaced linear array of four sensors, as shown in Fig. 2. From the above procedure, $\mathbf{C}_{k}$ and $\lambda_{k}$ for this four-sensor uniform linear array can be easily derived as

$$
\begin{gathered}
\mathbf{C}_{0}=\left[\begin{array}{l}
1 \\
0 \\
0 \\
0
\end{array}\right], \mathbf{C}_{1}=\left[\begin{array}{c}
-\rho_{1} \\
1 \\
0 \\
0
\end{array}\right], \mathbf{C}_{2}=\left[\begin{array}{c}
\frac{\rho_{1}^{2}-\rho_{2}}{1-\rho_{1}^{2}} \\
\frac{\rho_{1} \rho_{2}-\rho_{1}}{1-\rho_{1}^{2}} \\
1 \\
0
\end{array}\right], \\
\mathbf{C}_{3}=\left[\begin{array}{c}
\frac{-\rho_{1}^{3}+\rho_{1}^{2} \rho_{3}+2 \rho_{1} \rho_{2}-\rho_{1} \rho_{2}^{2}-\rho_{3}}{-2 \rho_{1}^{2}+2 \rho_{1}^{2} \rho_{2}-\rho_{2}^{2}+1} \\
\frac{-\rho_{1}^{2}-\rho_{1} \rho_{3}+\rho_{2}^{2}+\rho_{2}}{2 \rho_{1}^{2}-\rho_{2}-1} \\
\frac{\rho_{1}^{3}-\rho_{1}^{2} \rho_{3}-\rho_{1}+\rho_{1} \rho_{2}-\rho_{1} \rho_{2}^{2}+\rho_{2} \rho_{3}}{-2 \rho_{1}^{2}+2 \rho_{1}^{2} \rho_{2}-\rho_{2}^{2}+1} \\
1
\end{array}\right],
\end{gathered}
$$

where $\rho_{|i-j|}=\rho_{i j}=\rho_{j i}$ and $\rho_{0}=1$. The expressions of $\lambda_{k}$ are

$$
\begin{aligned}
& \lambda_{0}=1, \\
& \lambda_{1}=1-\rho_{1}^{2}, \\
& \lambda_{2}=\frac{-2 \rho_{1}^{2}+2 \rho_{1}^{2} \rho_{2}-\rho_{2}^{2}+1}{1-\rho_{1}^{2}}, \\
& \lambda_{3}=\frac{\rho_{1}^{4}-2 \rho_{1}^{3} \rho_{3}-2 \rho_{1}^{2} \rho_{2}^{2}+4 \rho_{1}^{2} \rho_{2}-3 \rho_{1}^{2}}{-2 \rho_{1}^{2}+2 \rho_{1}^{2} \rho_{2}-\rho_{2}^{2}+1} \\
& +\frac{\rho_{1}^{2} \rho_{3}^{2}-2 \rho_{1} \rho_{2}^{2} \rho_{3}+4 \rho_{1} \rho_{2} \rho_{3}+\rho_{2}^{4}-2 \rho_{2}^{2}-\rho_{3}^{2}+1}{-2 \rho_{1}^{2}+2 \rho_{1}^{2} \rho_{2}-\rho_{2}^{2}+1} .
\end{aligned}
$$

Equations (34) and (35) appear to be complex, especially when the order number is large. However, they are all in closed-form and can be directly calculated with the recursive formulas as $\rho_{i}$ are given. This approach provides a practical way to obtain an accurate solution for superdirectivity. The expressions of $\mathrm{C}_{k}$ and $\lambda_{k}$ with

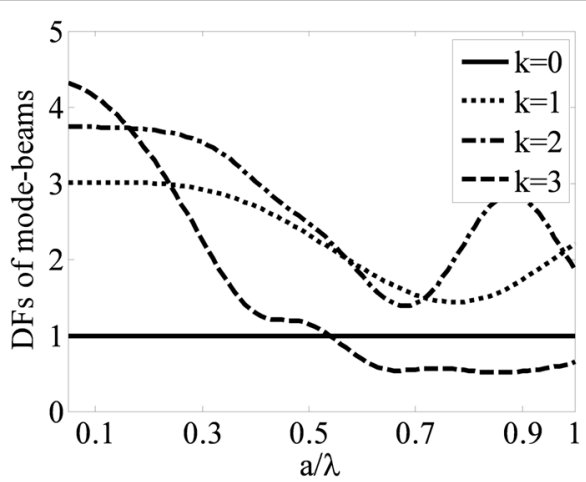

(a)

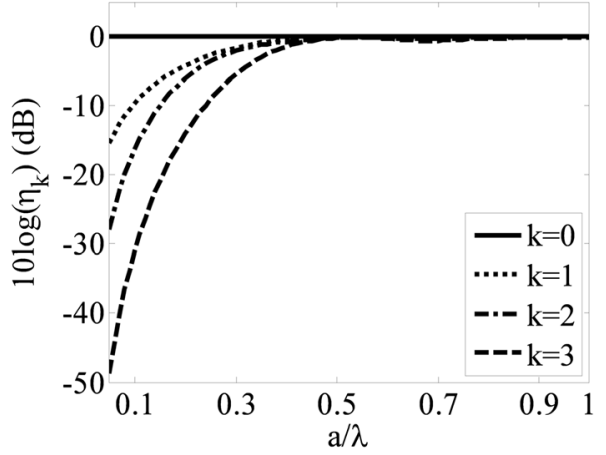

(b)

Fig. 7 DFs and levels of robustness of the Oth- to 3rd-order modified mode-beams for the six-sensor uniform circular array. a DFs. $\mathbf{b}$ Levels of robustness 


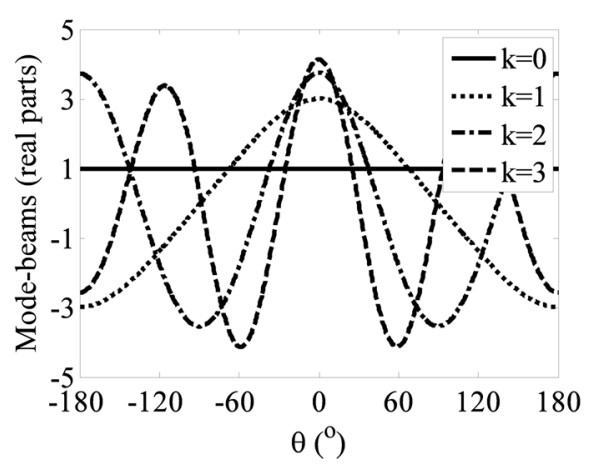

(a)

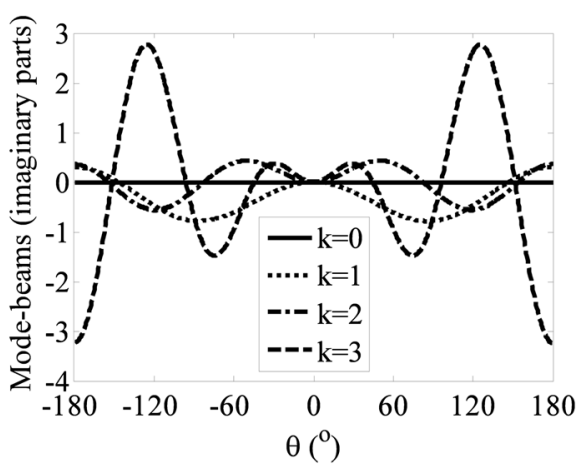

(b)

Fig. 8 The 0th- to 3rd-order modified mode-beams of the six-sensor uniform circular array at $a / \lambda=0.1$. a Real parts. $\mathbf{b}$ Imaginary parts

orders larger than 3 can be similarly derived but are not listed here for brevity.

The calculated DFs and levels of robustness $\left(10 \log \lambda_{k}\right)$ of the mode-beams are shown in Fig. 3a, b, respectively, in which $\left(\theta_{0}, \phi_{0}\right)=(0,0)$. When $d / \lambda<0.5$, both the DF and the error sensitivity increase with an increase in the order number $k$ and decrease with increasing $d / \lambda$. As $d / \lambda$ reaches 0 , the DF of the $k$ th-order mode-beam approaches $(2 k+1)$, and the total DF obtained by summation of the 0th- to $(N-1)$ th-order mode-beams is $N^{2}$. This property is consistent with

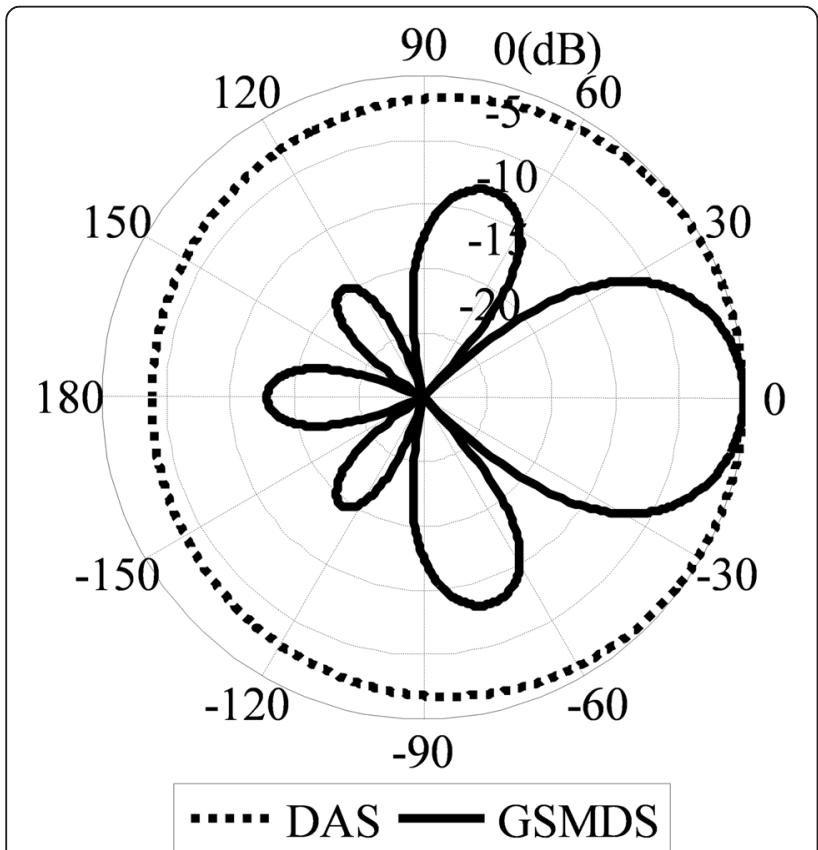

Fig. 9 Synthesized superdirective beampattern ( $\mathrm{Dl}=10.75 \mathrm{~dB}$ ) of the six-sensor uniform circular array compared with the conventional DAS beampattern $(\mathrm{DI}=1.39 \mathrm{~dB})$ at $a / \lambda=0.1$ the conclusions presented in [2] and shows significant potential in directivity improvement for linear arrays. However, the levels of robustness have no limit values and become infinite as $d / \lambda$ decreases to 0 . When $d / \lambda$ is larger than 0.5, the DFs and levels of robustness of different mode-beams tend to be equal, and their variances versus $d / \lambda$ are unclear. Note that the DF and level of robustness of the 0th-order mode-beam are constant in the entire frequency band, and the values are kept at 1 and $0 \mathrm{~dB}$, respectively.

Figure 4 shows the mode-beams with orders ranging from 0 to 3 . With the geometrical symmetry considered, calculations are only performed in the $x O z$ plane and $d / \lambda=0.1$. The synthesized overall beampatterns are shown in Fig. 5 in which the results of different models are compared, and inter-sensor spacing is differently set. It is clear that the DA [35] and GSMDS models provide the similar main lobe width, and both of them outperform the conventional beamforming (CBF, i.e., DAS beamforming) model. However, the sidelobes of the DA model, especially the opposite beampatterns, are higher than those of the GSMDS model because the DA model involves theoretical approximations. This type of distortions increases with the increasing frequency (c.f. Fig. 5a, b). Specifically, as shown in Fig. 5b, the beampattern produced by the DA model is largely modified when $d / \lambda=0.3$, and the opposite beampattern particularly becomes enormous. By contrast, the GSMDS model still works well.

\subsection{Circular arrays}

Circular arrays are desired in many circumstances because they have simple configurations, do not have left-right ambiguities, and can provide uniform beams along with $360^{\circ}$ azimuthal directions [4, 21, 22, 37]. A six-sensor uniform circular array with radius $a$ is 


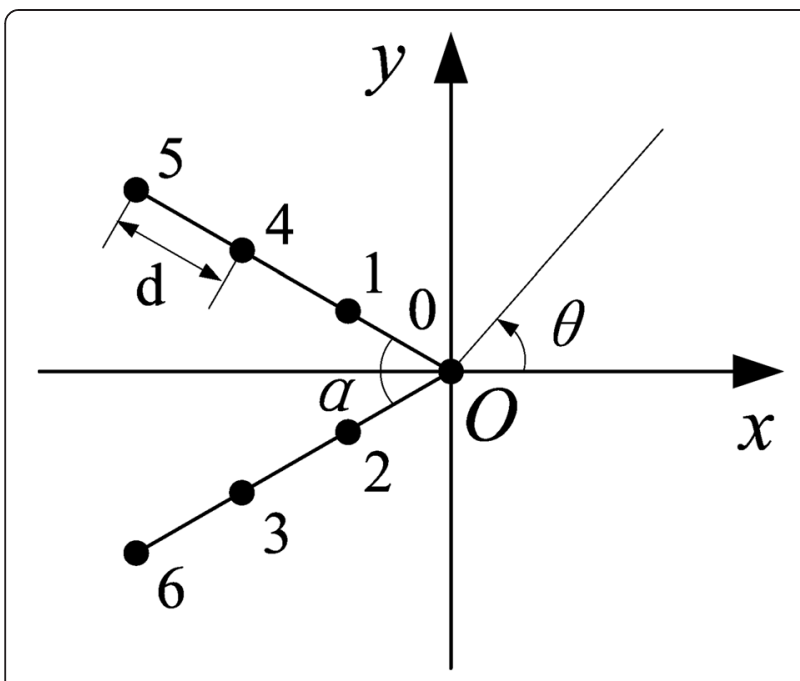

Fig. 10 Coordinates of a seven-sensor V-shaped array

considered, and its coordinates are shown in Fig. 6. The origin is deliberately not located at the center of the circle for the convenience provided in the decomposition and synthesis procedure. The numbers adjacent to the sensors in Fig. 6 indicate the processing order of GS transformation. The DFs and levels of robustness of all mode-beams can then be readily calculated with the use of Eqs. (23) and (22). However, an additional process is needed to clearly show the regularity of the GSMDS model for circular arrays. When $N$ is even, the mode-beams, DFs, and robustness parameters can be modified with the use of Eqs. (36) to (38):

$$
\hat{b}_{k}= \begin{cases}b_{k} & (k=0), \\ b_{2 k-1}+b_{2 k} & (k=1,2, \cdots, N / 2-1), \\ b_{2 k-1} & (k=N / 2)\end{cases}
$$

$$
\begin{gathered}
\hat{Q}_{k}= \begin{cases}Q_{k} & (k=0), \\
Q_{2 k-1}+Q_{2 k} & (k=1,2, \cdots, N / 2-1), \\
Q_{2 k-1} & (k=N / 2),\end{cases} \\
\eta_{k}= \begin{cases}\lambda_{k} & (k=0), \\
\left(\lambda_{2 k-1}+\lambda_{2 k}\right) / 2 & (k=1,2, \cdots, N / 2-1), \\
\lambda_{2 k-1} & (k=N / 2),\end{cases}
\end{gathered}
$$

and when $N$ is odd, the following equations are obtained:

$$
\begin{aligned}
& \hat{b}_{k}= \begin{cases}b_{k} & (k=0), \\
b_{2 k-1}+b_{2 k} & (k=1,2, \cdots,(N-1) / 2),\end{cases} \\
& \hat{Q}_{k}= \begin{cases}Q_{k} \\
Q_{2 k-1}+Q_{2 k} & (k=1,2, \cdots,(N-1) / 2),\end{cases} \\
& \eta_{k}= \begin{cases}\left.\lambda_{k}=0\right) \\
\left(\lambda_{2 k-1}+\lambda_{2 k}\right) / 2 & (k=1,2, \cdots,(N-1) / 2) .\end{cases}
\end{aligned}
$$

These equations are reasonable because of the symmetry between the $(2 k-1)$ th and $(2 k)$ th sensors, which is also utilized in [21]. Therefore, the total number of mode-beams will decrease to $(N / 2+1)$ (for even $N$ ) or $(N+1) / 2$ (for odd $N$ ) from $N$. The final synthesized beampattern and the total DF are unaffected by this modification, but the levels of robustness should be properly evaluated to keep them meaningful. The values of $\lambda_{2 k-1}$ and $\lambda_{2 k}$ have the same order of magnitude, and this means that the $(2 k-1)$ th- and $(2 k)$ th-order modebeams have similar robustness. At this point, their

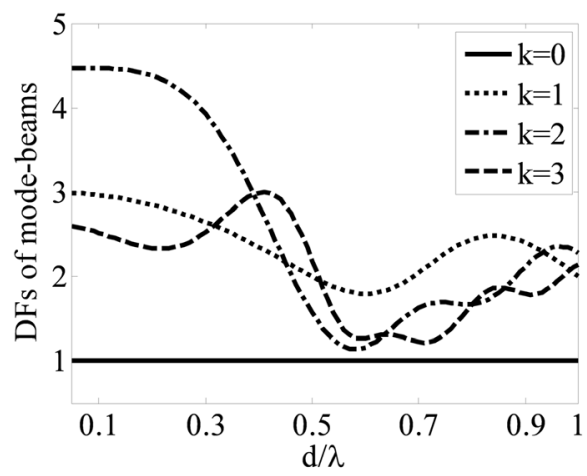

(a)

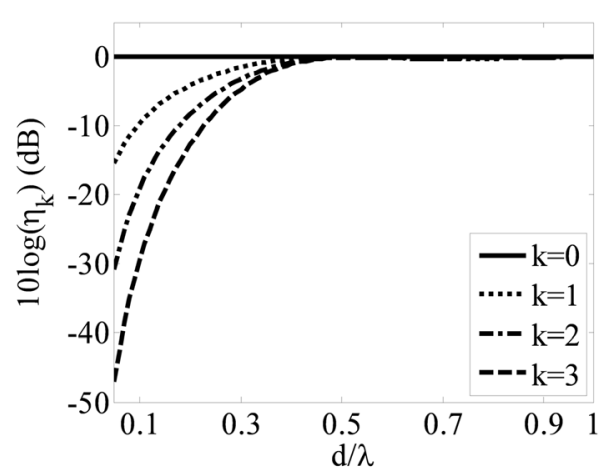

(b)

Fig. 11 DFs and levels of robustness of the Oth- to 3rd-order modified mode-beams for the seven-sensor V-shaped array. a DFs. b Levels of robustness 


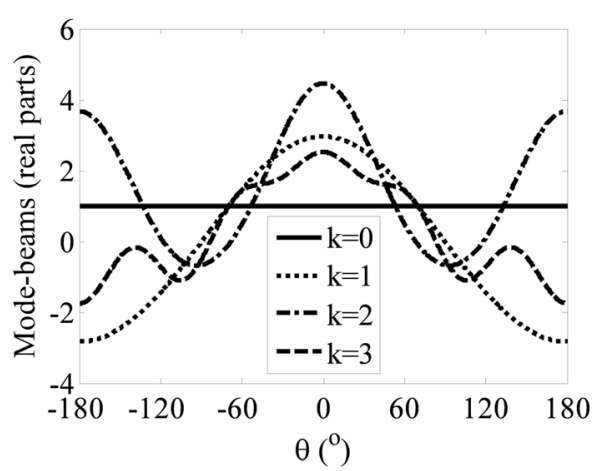

(a)

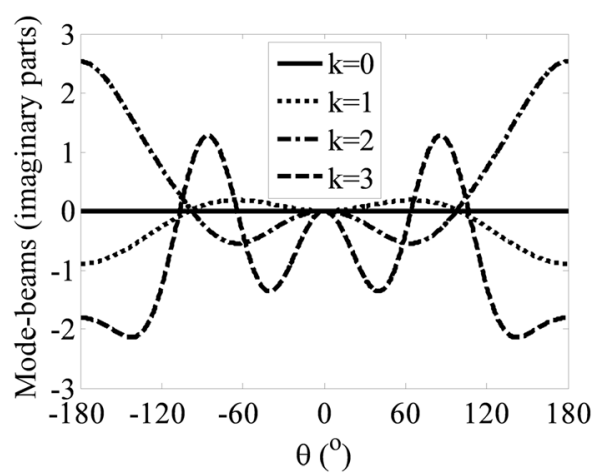

(b)

Fig. 12 The Oth- to 3rd-order modified mode-beams of the seven-sensor $V$-shaped array at $d / \lambda=0.1$. a Real parts. b Imaginary parts

average value $\eta_{k}$ is selected to measure the robustness of the $k$ th-order modified mode-beam.

The DFs and levels of robustness of the modified mode-beams are shown in Fig. 7a, b, respectively, and they depict the same behavior as that of linear arrays when $a / \lambda$ is small. Specifically, the larger the order number, the higher is the DF of this mode-beam, and the worse is the robustness. As $a / \lambda$ decreases, the DF also increases, and the mode-beam becomes error sensitive. When $a / \lambda$ is large, the levels of robustness almost overlap with one another, but the DFs become irregular.

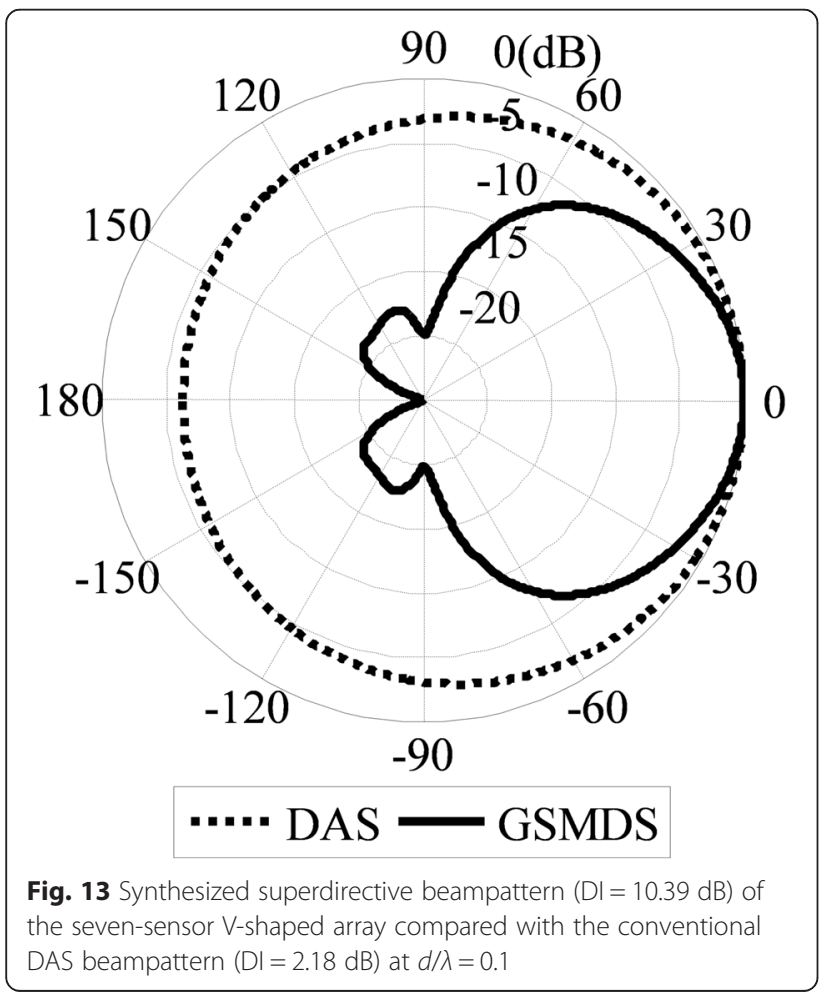

The 0th-order modified mode-beam still has a constant DF and robustness in the entire frequency band.

The 0th- to 3rd-order modified mode-beams for this six-sensor uniform circular array at $a / \lambda=0.1$ are shown in Fig. 8. Unlike the eigen-beams shown in [21], the imaginary parts of these mode-beams cannot be ignored (see Fig. 8b). As shown in Fig. 9, the synthesized final superdirective beampattern using all mode-beams is actually the same as the final result using all eigen-beams in [21], which shows apparent performance improvement compared with the conventional DAS beampattern. Note that the EBDS model appears to be simpler for circular arrays than the GSMDS model, but it cannot be applied to other arrays. The superiority of the GSMDS model over the EBDS one is clear.

\section{3 "V"-shaped arrays}

The V-shaped arrays have good potential to be used in practical applications, such as in underwater acoustic communication and navigation systems for small autonomous vehicles. They can also effectively show the advantages of the GSMDS model. A seven-sensor Vshaped array is shown in Fig. 10. It consists of two foursensor uniform linear arrays with a common sensor. These two linear arrays are axisymmetric with respect to the $x$-axis. The opening angle $\alpha$ is set to $60^{\circ}$.

The DFs and levels of robustness shown in Fig. 11a, b, respectively, were modified with the use of Eqs. (40) and (41), respectively, and the number of mode-beams was changed to four. Note that the DFs do not regularly change with $d / \lambda$ or the order number, but the levels of robustness still show the same behavior as those of the linear and circular arrays. Figure 12 shows the 0th- to 3rd-order modified mode-beams for the seven-sensor Vshaped array at $d / \lambda=0.1$. The synthesized superdirective beampattern using these mode-beams is compared with 


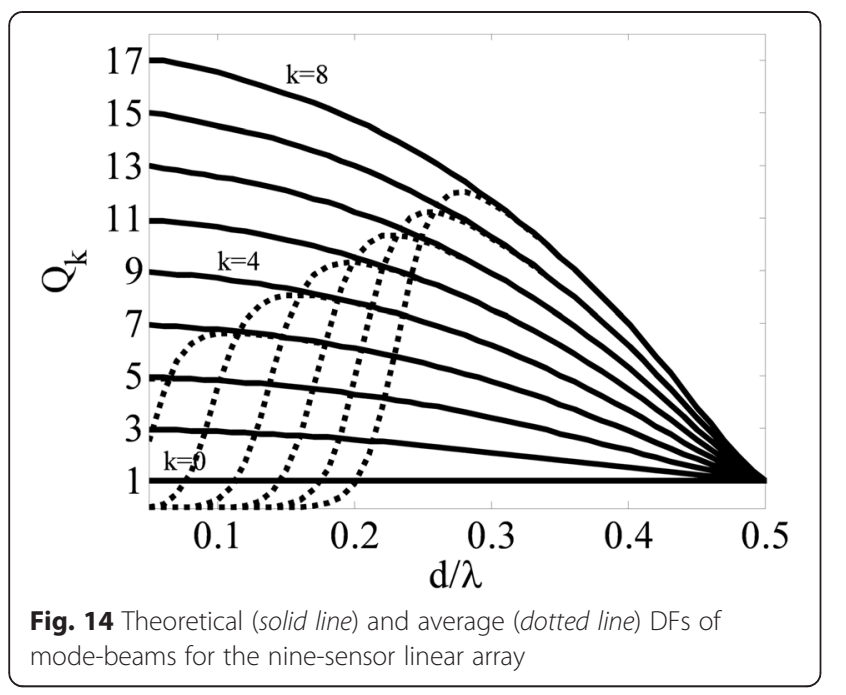

the conventional DAS beampattern in Fig. 13. The performance enhancement is also clear.

These analyses indicate that the GSMDS model can accurately decompose the optimal solutions of superdirectivity into different sub-solutions for arrays of different shapes. When it is applied to the above three array types (not limited to these arrays), the robustness property of the mode-beams with high orders clearly becomes error sensitive. Therefore, the reduced-rank technique can be readily used to synthesize robust high-order superdirective beampatterns.

\section{Comparisons with the DAS and MVDR methods}

Some detailed comparisons between the GSMDS method and some widely used methods are presented in the following simulations, so that the advantages of the proposed method will be clearer.

We regard a nine-sensor linear array as an example. The sensor gain and phase errors, termed $g$ and $\psi$,

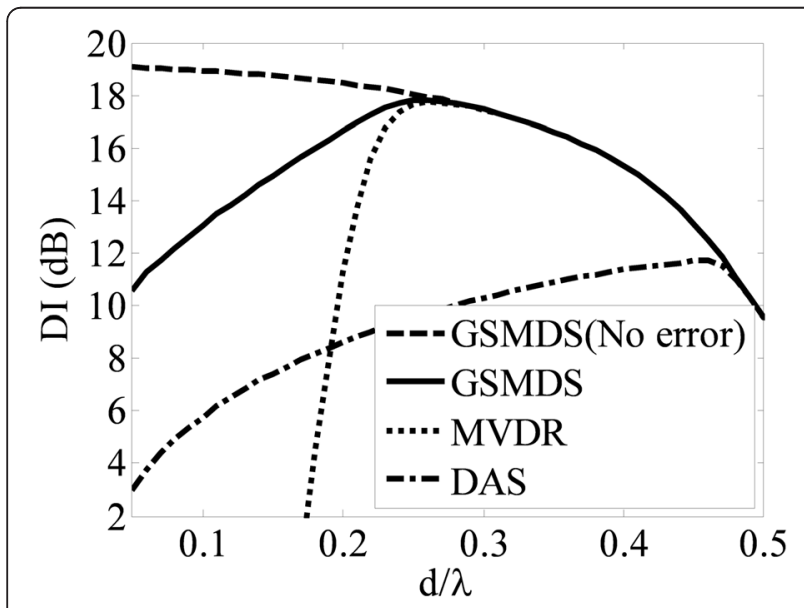

Fig. 15 Dls of different methods for the nine-sensor linear array

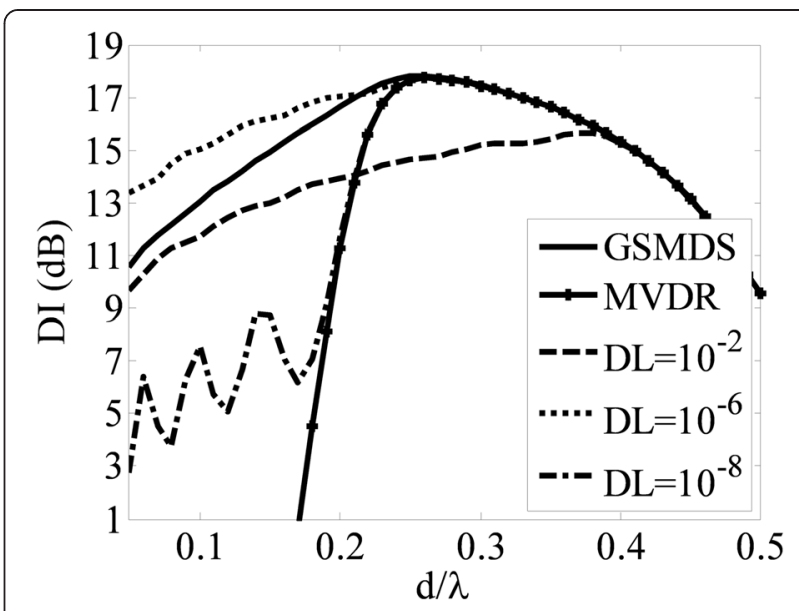

Fig. 16 Average Dls of different methods for the nine-sensor linear array

respectively, are assumed to be statistically independent, zero mean, Gaussian random variables, and their variances are set to $\sigma_{g}^{2}=\sigma_{\psi}^{2}=10^{-6}$.

The average values of actual DFs of the mode-beams calculated using Eq. (32) are shown in Fig. 14 (dotted lines), in which the different mode-beams show different sensitivities to errors at this level of errors. Specifically, the mode-beams with orders lower than 3 are all sufficiently robust. Their average DFs show no changes in the given frequency band, whereas the average DFs of the 3th- to 8th-order mode-beams decrease at some frequencies, called "critical frequencies". The smaller the order, the lower is the critical frequency. This is because the lower-order modebeams correspond to smaller frequencies when the required robustness level is the same, as shown in Fig. 3 b. It is clear that the high-order mode-beams in Fig. 14 do not contribute to the total DF in some frequency ranges, and these mode-beams can be truncated, leading to the improved robustness. If better superdirectivity is required to be obtained in practice, the maximum order of the retained mode-beams is unnecessarily selected to be large.

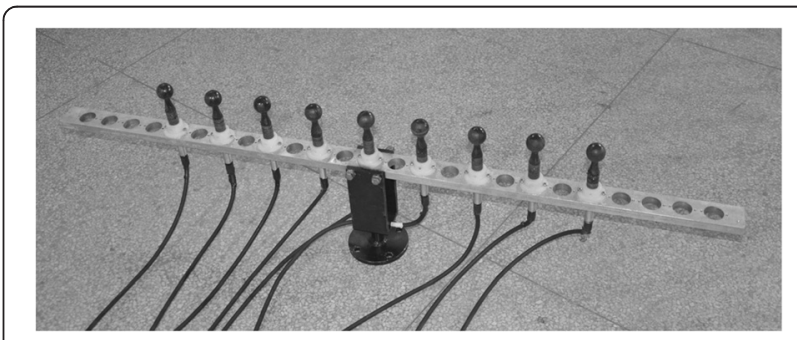

Fig. 17 Experimental uniform linear array of nine omnidirectional hydrophones 

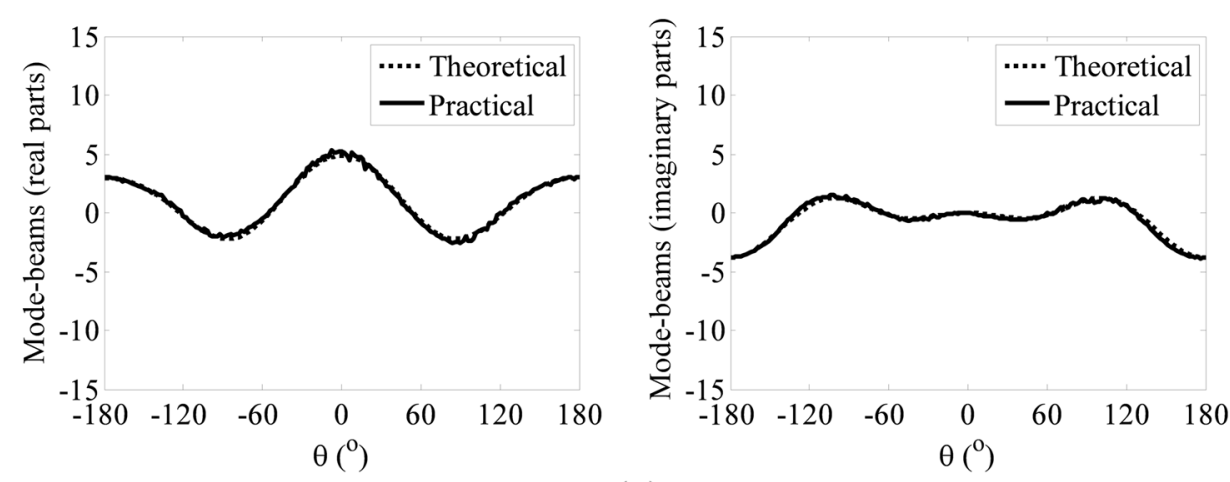

(a)
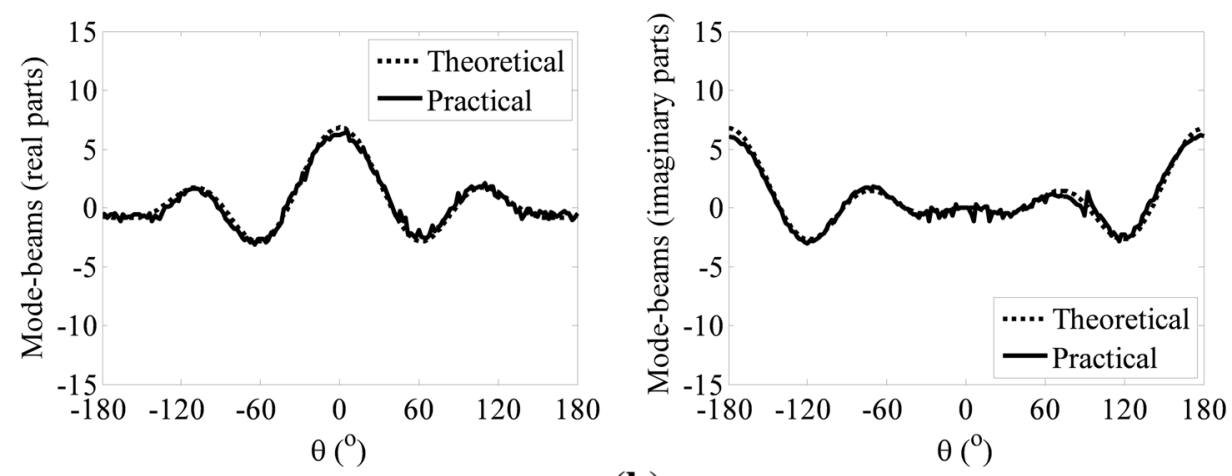

(b)
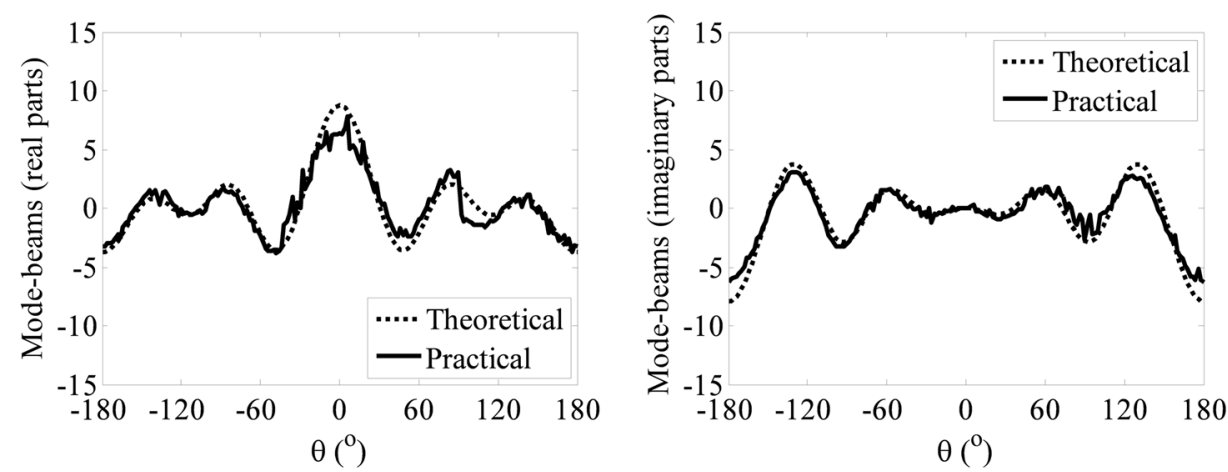

(c)
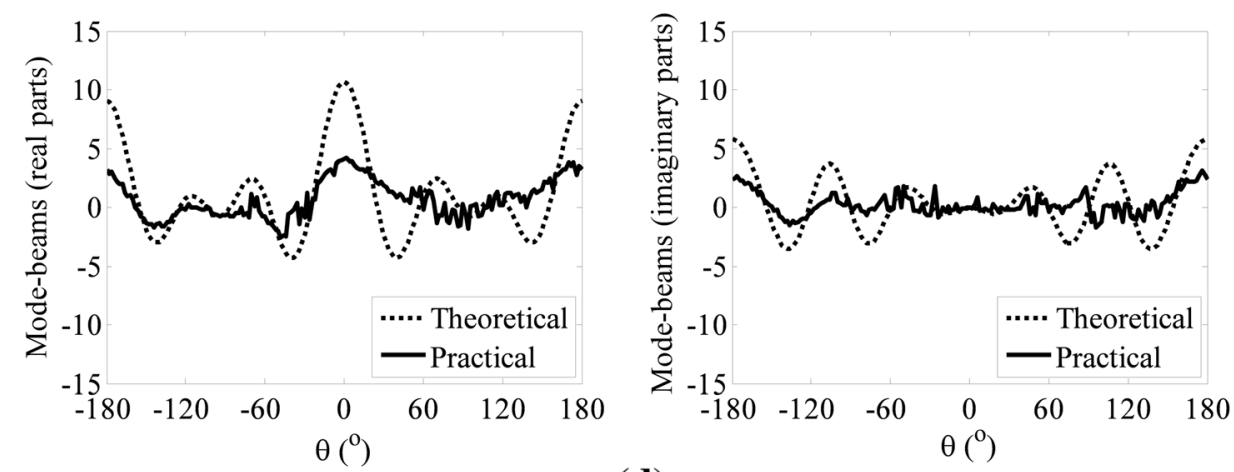

(d)

Fig. 18 Theoretical and practical mode-beams at $f=1350 \mathrm{~Hz}$. a $k=2$. b $k=3 . \mathbf{c} k=4$. d $k=5$ 


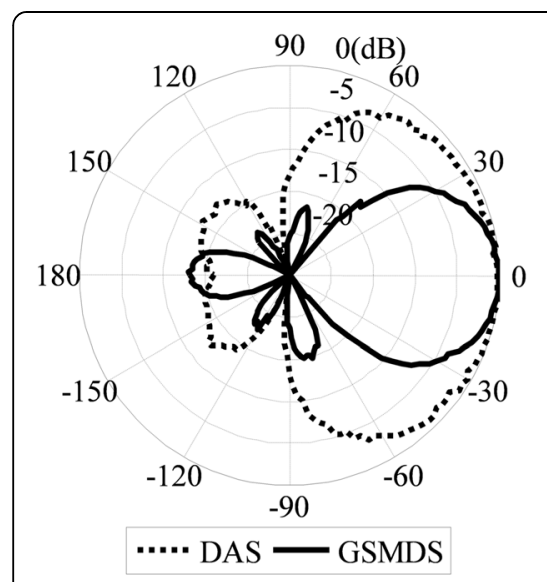

(a)

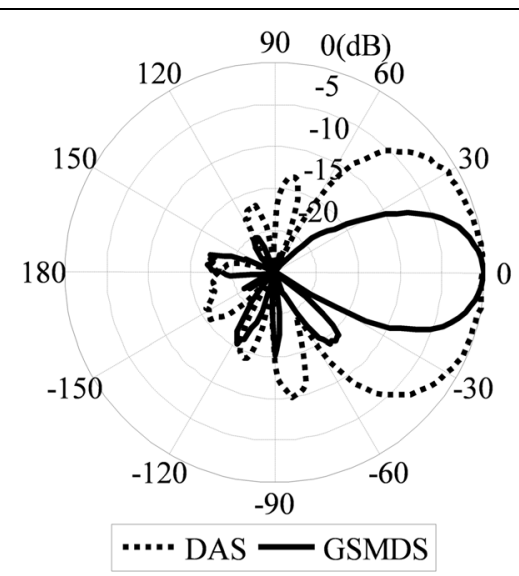

(b)

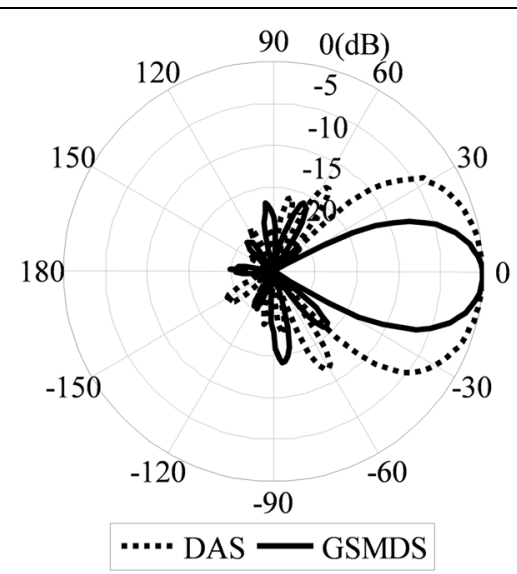

(c)

Fig. 19 Synthesized experimental superdirective beampatterns for the nine-hydrophone linear array compared with conventional DAS beampatterns. a $f=1350 \mathrm{~Hz}(d / \lambda=0.09), K=3 . \mathbf{b} f=3000 \mathrm{~Hz}(d / \lambda=0.2), K=6 . \mathbf{c} f=5000 \mathrm{~Hz}(d / \lambda=0.33), K=8$

The average DIs of this array achieved using different methods are shown in Fig. 15 by considering the same level of errors $\left(\sigma_{g}^{2}=\sigma_{\psi}^{2}=10^{-6}\right)$. The DAS method is the most robust method, but its directivity is the lowest. By contrast, the minimum variance distortionless response (MVDR) method can provide the highest directivity, but its robustness is the worst. The average DI of the MVDR method degrades at approximately $d / \lambda=0.26$ and is even less than that of the DAS method when $d / \lambda<0.19$. The theoretical DI of the GSMDS method without any errors, which is obtained by summing the theoretical DFs of mode-beams shown in Fig. 14, is equal to that of the MVDR method. Furthermore, at $d / \lambda=0.26$, the average DI of the GSMDS method, which is achieved by summing the average DFs of mode-beams in Fig. 14, starts to decrease but with a slower velocity. Compared with the MVDR method, the achieved average DI of the GSMDS method can reach $16.3 \mathrm{~dB}$ at $d / \lambda=0.19$, which is also

Table 1 Performance measures of practical beampatterns

\begin{tabular}{llll}
\hline Method & DI $(d B)$ & HPBW $\left(^{\circ}\right)$ & SL $(d B)$ \\
\hline$f=1350 \mathrm{~Hz}(d / \lambda=0.09)$ & & & \\
DAS & 5.80 & 118.74 & -12.46 \\
GSMDS $(K=3)$ & 11.92 & 48.05 & -12.98 \\
$f=3000 \mathrm{~Hz}(d / \lambda=0.2)$ & & & \\
DAS & 8.63 & 80.70 & -9.99 \\
GSMDS $(K=6)$ & 15.01 & 31.41 & -14.00 \\
$f=5000 \mathrm{~Hz}(d / \lambda=0.33)$ & & & -11.65 \\
DAS & 11.07 & 59.50 & -14.16 \\
GSMDS $(K=8)$ & 15.57 & 28.68 & \\
\hline
\end{tabular}

higher than that of the DAS method. When the frequency is lower, the DI of the GSMDS method is still larger than that of the DAS and MVDR methods owing to the contribution of the robust lower-order mode-beams.

\section{Comparisons with the regularization-based methods}

As presented previously, the type of regularizationbased methods, specifically the DL method, is extensively used in practice and can be selected as a typical example to compare with the proposed method. The average DIs obtained using these two methods are shown in Fig. 16.

It is observed that the average DI of the DL method is larger than that of the MVDR method in the low-frequency band, which indicates that adding a regularization parameter (i.e., DL value) effectively improves robustness. However, if the selected regularization parameter is large, then the beamformer is over-robust and the DI is theoretically low. If the regularization parameter selected is small, then robustness is not sufficiently good to lead to an exceedingly low actual DI. In Fig. 16, the average DIs of $\mathrm{DL}=10^{-2}$ (large) and $\mathrm{DL}=10^{-8}$ (small) in the lowfrequency range are lower than those of the GSMDS method, whereas the obtained DI for $\mathrm{DL}=10^{-6}$ is larger than that of the GSMDS method. It is clear that the regularization-based methods will perform better than the GSMDS method when the regularization parameter is appropriately selected (e.g., $\mathrm{DL}=10^{-6}$ in this example), but the appropriate parameter is not easily determined in many practical applications because the level of actual errors is always unknown so that the final results will be always degraded in some frequency bands. By contrast, the performance of the GSMDS 
method is determined when the actual level of errors is given, and it will not change with other parameters. In other words, although the regularization-based methods can work better than the GSMDS method, they also have the great possibility to give worse results in some frequency ranges over the proposed method in practical applications. For a conservative purpose, the GSMDS method will be preferred to be applied in practice because it has the determined performance and takes no risks of giving worse results in comparison with the regularization-based methods.

\section{Experimental results}

An experimental uniform linear array consisting of nine omnidirectional hydrophones was prepared. This array is shown in Fig. 17, and the inter-sensor spacing is $10 \mathrm{~cm}$. The data acquired in an anechoic water tank were used for experimental verification. The signals employed in the experiment were rectangular-windowmodulated single-frequency continuous-wave pulses, and their frequencies were 5000, 3000, and $1350 \mathrm{~Hz}$. Figure 18 shows the theoretical and practical modebeams with orders ranging from 2 to 5 at a frequency of $1350 \mathrm{~Hz}(d / \lambda=0.09)$. The mode-beams with orders smaller than 4 clearly agree well with the corresponding theoretical ones. Because of the errors from hydrophone gain and phase mismatches, sensor position perturbations, and structural scattering, all the high-order modebeams are corrupted, and distortions increase with an increase in the order number. This condition is consistent with the above-presented analyses on $\lambda_{k}$. Although the 0th- and 1st-order mode-beams, as well as the 6thto 8 th-order ones, are not shown here, their properties are self-evident. The mode-beams at $3000 \mathrm{~Hz}(d / \lambda=0.2)$ and $5000 \mathrm{~Hz}(d / \lambda=0.33)$ also exhibit similar behavior, but they are not shown here for brevity.

The above analyses indicate that the overall practical beampatterns at these three frequencies can be easily synthesized. Figure 19 shows these superdirective beampatterns, and Table 1 lists their performance measures, including DIs, half-power beamwidths (HPBWs), and sidelobe levels (SLs). The DI values were calculated with the assumptions that the beampatterns had rotational symmetry, and the noise field was isotropic. The SL was defined as the maximum level of sidelobes. The frequency is $1350 \mathrm{~Hz}$, so the practical mode-beams with orders larger than 3 are unusable because of their error sensitivities. Therefore, a reduced-rank beampattern is synthesized with the use of only the 0th- to 3rd-order mode-beams $(K=3)$. The measured SL is almost the same as the conventional DAS beampattern, but the HPBW decreases to $48.05^{\circ}$ unlike the conventional DAS beamwidth at approximately $118.74^{\circ}$. Because the main lobe is pointed toward the endfire direction, the DF is improved by approximately a factor of four in this particular case and $6 \mathrm{~dB}$ for the DI. For high frequencies, e.g., $3000 \mathrm{~Hz}$, the 4th- to 6th-order mode-beams $(K=6)$ are also usable, and the DI of the final superdirective beampattern is improved by approximately $6 \mathrm{~dB}$ over its conventional DAS counterpart. When the frequency increases to $5000 \mathrm{~Hz}$, all mode-beams $(K=8)$ are robust against errors and can be used to synthesize the superdirective beampattern. However, the DI enhancement is approximately $4.5 \mathrm{~dB}$, which is smaller than those in the two above cases. Both the superdirective beampatterns have lower SLs and narrower HPBWs than the conventional DAS beampatterns when the frequencies are 3000 and $5000 \mathrm{~Hz}$, a result indicating better performance over the DAS method.

It is found that the effect of actual errors on mode-beams for this experimental linear array is similar to that shown in Fig. 14, although the actual errors of the experimental linear array are not only from the sensor gain and phase. According to the analyses in the "Comparisons with the DAS and MVDR methods" section, the average DFs of mode-beams with orders smaller than 4 show good performance at $1350 \mathrm{~Hz}(d / \lambda=0.09)$, meaning the appropriate maximum order is 3 at this level of errors. From Fig. 14, it is also inferred that the appropriate maximum order will be 5 and 8 when the frequencies are $3000 \mathrm{~Hz}(d / \lambda=0.2)$ and $5000 \mathrm{~Hz}$ $(d / \lambda=0.33)$, respectively, if the actual errors are independent of the frequency. The conclusions for the cases at $1350 \mathrm{~Hz}(d / \lambda=0.09)$ and $5000 \mathrm{~Hz}(d / \lambda=0.33)$ are approximately right, whereas the case at $3000 \mathrm{~Hz}$ $(d / \lambda=0.2)$ shows some deviations in experimental results because of effects of other errors.

As presented previously, the highest order of satisfactory mode-beams has to be selected with special attention, and this selection deserves a final consideration. If the level of actual errors of a practical array can be precisely estimated, then the highest order can be easily determined with the use of computer simulations, and the truncation of unsatisfactory high-order mode-beams can be conveniently conducted. When the information of actual errors cannot be known, an ad hoc experimental measurement is required to determine the highest order, which can also be effectively used during truncation, as is the case in this study.

\section{Conclusions}

This study presents a general superdirectivity model for arbitrary sensor arrays. Because the noise correlation coefficients between sensors can be easily determined in the isotropic noise field, all the solutions of superdirectivity have been accurately expressed in full closed-form based on the GS orthogonalization scheme with the use of the frequency and array geometric parameters. The results indicate that theoretical approximations are not 
involved in this model, meaning this model provides an effective mechanism to achieve high-order superdirectivity. The beamforming process is simplified by decomposition of the optimal beampattern and maximum DF into mode-beams and their associated DFs to significantly facilitate the implementation of superdirectivity. All of these results are directly derived from the optimal solutions of superdirectivity, so the GSMDS method can be applied to sensor arrays with arbitrary geometries, and no a priori knowledge of errors are required. Design examples for three different arrays show that as the order number increases, the robustness of mode-beams decreases. Therefore, robust superdirective beampatterns should be synthesized with the use of a reduced-rank treatment. The performance of this technique is demonstrated with simulations and real-data experiments. Further work can focus on the error control for high-order mode-beams to further improve the practically achievable superdirectivity. An in-depth study of applying this model to other complex arrays, such as volumetric arrays, will also be a main topic in the further.

\section{Competing interests}

The authors declare that they have no competing interests.

\section{Acknowledgements}

This work was supported by the National Natural Science Foundation of China (Grant Nos. 11274253 and 60901076) and the Fundamental Research Funds for the Central Universities (Grant No. 3102014JCQ01008).

Received: 9 February 2015 Accepted: 14 July 2015

Published online: 02 August 2015

\section{References}

1. HL Van Trees, Optimum Array Processing: Part N of Detection, Estimation, and Modulation theory (John Wiley \& Sons, Inc, New York, 2002), pp. 59-71. 274-289, 439-443

2. Al Uzkov, An approach to the problem of optimum directive antenna design. Compt. Rend. Acad. Sci. U.R.S.S. 53, 35-38 (1946)

3. JB Franklin, Superdirective Receiving Arrays for Underwater Acoustics Application (Defence Research Establishment Atlantic, Dartmouth, Nova Scotia, 1997)

4. Q-C Zhou, H Gao, H Zhang, F Wang, Robust superdirective beamforming for HF circular receive antenna arrays. Prog. Electromagn. Res. 136, 665-679 (2013)

5. E Shamonina, L Solymar, Maximum directivity of arbitrary dipole arrays. IET Microwaves, Antennas Propagation 9, 101-107 (2015)

6. R Berkun, I Cohen, J Benesty, Combined beamformers for robust broadband regularized superdirective beamforming. IEEE/ACM Trans. Audio Speech Lang. Process. 23, 877-886 (2015)

7. BD Carlson, Covariance matrix estimation errors and diagonal loading in adaptive arrays. IEEE Trans. Aero. Elec. Sys. 24, 397-401 (1988)

8. H Cox, R Zeskind, M Owen, Robust adaptive beamforming. IEEE Trans. Acoust. Speech Signal Process. 35, 1365-1376 (1987)

9. MR Bai, C-C Chen, Regularization using Monte Carlo simulation to make optimal beamformers robust to system perturbations. J. Acoust. Soc. Am. $135,2808-2820(2014)$

10. S Doclo, M Moonen, Design of broadband beamformers robust against gain and phase errors in the microphone array characteristics. IEEE Trans. Signal Process. 51, 2511-2526 (2003)

11. M Crocco, A Trucco, The synthesis of robust broadband beamformers for equally-spaced linear arrays. J. Acoust. Soc. Am. 128, 691-701 (2010)

12. A Trucco, M Crocco, Design of an optimum superdirective beamformer through generalized directivity maximization. IEEE Trans. Signal Process. 62, 6118-6129 (2014)

13. S Doclo, M Moonen, Superdirective beamforming robust against microphone mismatch. IEEE Trans Audio Speech Lang Process 15, 617-631 (2007)
14. M Crocco, A Trucco, Design of robust superdirective arrays with a tunable tradeoff between directivity and frequency-invariance. IEEE Trans. Signal Process. 59, 2169-2181 (2011)

15. A Trucco, F Traverso, M Crocco, Robust superdirective end-fire arrays (Proceedings of OCEANS 2013, Bergen, Norway, 2013), pp. 1-6

16. H Cox, RM Zeskind, T Kooij, Practical supergain. IEEE Trans. Acoust. Speech Signal Process. 34, 393-398 (1986)

17. DJ Schmidlin, Directionality of generalized acoustic sensors of arbitrary order. J. Acoust. Soc. Am. 121, 3569-3578 (2007)

18. BA Cray, AH Nuttall, Directivity factors for linear arrays of velocity sensors. J. Acoust. Soc. Am. 110, 324-331 (2001)

19. BA Cray, VM Evora, AH Nuttall, Highly directional acoustic receivers. J. Acoust. Soc. Am. 113, 1526-1532 (2003)

20. GL D'Spain, JC Luby, GR Wilson, RA Gramann, Vector sensors and vector sensor line arrays: comments on optimal array gain and detection. J. Acoust. Soc. Am. 120, 171-185 (2006)

21. YL Ma, YX Yang, ZY He, KD Yang, C Sun, YM Wang, Theoretical and practical solutions for high-order superdirectivity of circular sensor arrays. IEEE Trans. Ind. Electron. 60, 203-209 (2013)

22. Y Wang, YX Yang, YL Ma, ZY He, Robust high-order superdirectivity of circular sensor arrays. J. Acoust. Soc. Am. 136, 1712-1724 (2014)

23. AT Parsons, Maximum directivity proof for three-dimensional arrays. J. Acoust. Soc. Am. 82, 179-182 (1987)

24. M Uzsoky, L Solymar, Theory of super-directive linear arrays. Acta Phys. Acad. Sci. Hungary 6, 185-205 (1956)

25. RJ Urick, Principles of underwater sound (McGraw-Hill, New York, 1983), p. 42

26. F Ling, D Manolakis, JG Proakis, A recursive modified Gram-Schmidt algorithm for least-squares estimation. IEEE Trans. Acoust. Speech Signal Process. 34, 829-836 (1986)

27. K Gerlach, FF Kretschmer, Convergence properties of Gram-Schmidt and SMI adaptive algorithms. IEEE Trans. Aero. Elec. Sys. 26, 44-56 (1990)

28. K Gerlach, FF Kretschmer, Convergence properties of Gram-Schmidt and SMI adaptive algorithms. II, IEEE Trans. Aero. Elec. Sys. 27, 83-91 (1991)

29. IN Sahalos, Orthogonal Methods for Array Synthesis: Theory and the ORAMA Computer Tool (Wiley, London, 2006), pp. 69-72

30. S Enomoto, Y Ikeda, S Ise, S Nakamura, Optimization of Loudspeaker and Microphone Configurations for Sound Reproduction System Based on Boundary Surface Control Principle (Proceedings of the 20th International Congress on Acoustics, ICA 2010, Sydney, Australia, 2010), pp. 1-7

31. JWR Griffiths, JE Hudson, An introduction to adaptive processing in a passive sonar system, in Aspects of signal processing, ed. by G Tacconi (Springer, Netherlands, 1977), pp. 299-308. Vol. 33-1

32. SA Schelkunoff, A mathematical theory of linear arrays. Bell Syst. Tech. J. 22, 80-107 (1943)

33. RL Pritchard, Optimum directivity patterns for linear point arrays. J. Acoust. Soc. Am. 25, 879-891 (1953)

34. RL Pritchard, Maximum directivity index of a linear point array. J. Acoust. Soc. Am. 26, 1034-1039 (1954)

35. VF Humphrey, PC Hines, V Young, Experimental analysis of the performance of a superdirective line array, in Proceedings of the Seventh European Conference on Underwater Acoustics (Delft, Holland, 2004), pp. 1033-1038

36. A Trucco, F Traverso, M Crocco, Broadband performance of superdirective delay-and-sum beamformers steered to end-fire. J. Acoust. Soc. Am. 135, EL331-EL337 (2014)

37. Y Wang, YX Yang, YL Ma, ZY He, YK Liu, Broadband pattern synthesis for circular sensor arrays. J. Acoust. Soc. Am. 136, EL153-EL158 (2014) 